\title{
Gradual pressure-induced enhancement of magnon excitations in CeCoSi
}

\author{
S. E. Nikitin, ${ }^{1,2,{ }^{*}}$ D. G. Franco $\odot,{ }^{1,3}$ J. Kwon, ${ }^{1}$ R. Bewley, ${ }^{4}$ A. Podlesnyak $\odot,{ }^{5}$ A. Hoser, ${ }^{6}$ M. M. Koza, ${ }^{7}$ C. Geibel, ${ }^{1}$ \\ and O. Stockert ${ }^{1}$ \\ ${ }^{1}$ Max Planck Institute for Chemical Physics of Solids, D-01187 Dresden, Germany \\ ${ }^{2}$ Institut für Festkörper- und Materialphysik, Technische Universität Dresden, D-01069 Dresden, Germany \\ ${ }^{3}$ Centro Atómico Bariloche and Instituto Balseiro, Comisión Nacional de Energía Atómica (CNEA), \\ Universidad Nacional de Cuyo, Consejo Nacional de Investigaciones Científicas y Técnicas (CONICET), \\ Av. E. Bustillo 9500, R8402AGP San Carlos de Bariloche, Río Negro, Argentina \\ ${ }^{4}$ ISIS Facility, STFC Rutherford Appleton Laboratory, Harwell Campus, Didcot OX11 OQX, United Kingdom \\ ${ }^{5}$ Neutron Scattering Division, Oak Ridge National Laboratory, Oak Ridge, Tennessee 37831, USA \\ ${ }^{6}$ Helmholtz-Zentrum Berlin für Materialien und Energie, D-14109 Berlin, Germany \\ ${ }^{7}$ Institut Laue-Langevin, F-38042 Grenoble Cedex 9, France
}

(Received 1 April 2020; revised manuscript received 6 May 2020; accepted 19 May 2020; published 17 June 2020)

\begin{abstract}
$\mathrm{CeCoSi}$ is an intermetallic antiferromagnet with a very unusual temperature-pressure phase diagram: at ambient pressure it orders below $T_{\mathrm{N}}=8.8 \mathrm{~K}$, while application of hydrostatic pressure induces a new magnetically ordered phase with exceptionally high transition temperature of $\sim 40 \mathrm{~K}$ at $1.5 \mathrm{GPa}$. We studied the magnetic properties and the pressure-induced magnetic phase of CeCoSi by means of elastic and inelastic neutron scattering (INS) and heat capacity measurements. At ambient pressure CeCoSi orders into a simple commensurate AFM structure with a reduced ordered moment of only $m_{\mathrm{Ce}}=0.37(6) \mu_{\mathrm{B}}$. Specific heat and low-energy INS indicate a significant gap in the low-energy magnon excitation spectrum in the antiferromagnetic phase, with the CEF excitations located above $10 \mathrm{meV}$. Hydrostatic pressure gradually shifts the energy of the magnon band towards higher energies and the temperature dependence of the magnons measured at $1.5 \mathrm{GPa}$ is consistent with the phase diagram. Moreover, the CEF excitations are also drastically modified under pressure.
\end{abstract}

DOI: $10.1103 /$ PhysRevB.101.214426

\section{INTRODUCTION}

Ce-based intermetallic compounds represent a rich playground for exploration of quantum critical phenomena [1-4]. The ground state of these materials originates quite often from a competition between RKKY interaction and Kondo screening, which tend to create long-range magnetically ordered and nonmagnetic heavy-fermion states, respectively. The delicate balance between RKKY and Kondo effects can be quite easily tuned by external tuning parameters, e.g., composition, uniaxial or hydrostatic pressure, magnetic field, etc. Usually, application of hydrostatic pressure enhances the coupling between the conduction electrons and the localized Ce moments $J_{\text {cf }}$, and, therefore, drastically increases the strength of the Kondo effect $\left[T_{\mathrm{K}} \propto \exp \left(-\frac{1}{2 J_{\mathrm{cf}}}\right)\right]$ leading to a reduced magnetic ordering temperature and shifting the ground state of the material closer towards a nonmagnetic heavy-fermion state [5-8].

\footnotetext{
*nikitin@cpfs.mpg.de
}

Published by the American Physical Society under the terms of the Creative Commons Attribution 4.0 International license. Further distribution of this work must maintain attribution to the author(s) and the published article's title, journal citation, and DOI. Open access publication funded by the Max Planck Society.
However, in several recent works it was shown that $\mathrm{CeCoSi}$ represents an intriguing counterexample to this paradigm $[9,10]$. This material crystallizes in the tetragonal $\mathrm{CeFeSi}$ structure (space group $P 4 / \mathrm{nmm}$ ) and the cerium moments order antiferromagnetically below the $T_{\mathrm{N}}=8.8 \mathrm{~K}[11,12]$. Results of powder neutron diffraction measurements revealed a commensurate antiferromagnetic structure in isostructural $\mathrm{CeCoGe}$ with a simple antiferromagnetic stacking of FM Ce planes along the $c$ axis [13], but the information about the magnetic structure of $\mathrm{CeCoSi}$ is absent to the best of our knowledge. Resistivity measurements under hydrostatic pressure [9] have shown that the application of rather moderate pressure of only $\sim 0.6 \mathrm{GPa}$ induces a new magnetically ordered phase with exceptionally high transition temperature $T_{\mathrm{c}} \approx 40 \mathrm{~K}$ (see the phase diagram in Fig. 9). The pressureinduced phase has a dome shape and the $T_{\mathrm{c}}$ changes only slightly up to $\sim 1.7 \mathrm{GPa}$, whereas upon further pressure increase $T_{\mathrm{c}}$ gets rapidly suppressed and a quantum critical point, characterized by a divergence of resistivity parameters $A$ and $\rho_{0}$, was found at $\sim 2.2 \mathrm{GPa}$ [9]. A nonmagnetic Fermi-liquid state was observed at higher pressures.

In a recent study on single crystals, a very weak anomaly was observed in the specific heat and in the susceptibility at about $12 \mathrm{~K}$ and was proposed to be quadrupolar order [14]. Subsequent NMR and NQR results at high pressure indicate that the high- $T$ transition under pressure is a weak structural transition [15]. Its primary order parameter was also 
proposed to be an antiferroquadrupolar one. However $\mathrm{Ce}^{3+}$ is a Kramers ion, and in solids its $J=5 / 2$ multiplet is split into 3 Kramers doublets, which do not bear a quadrupolar degree of freedom. A quadrupolar order is then only possible by mixing excited CEF doublets, which requires the excited CEF states to be at low energy, of the order of the quadrupolar ordering temperature. However, preliminary results indicated the $\mathrm{CEF}$ splitting to be much larger, larger than $100 \mathrm{~K}$ [9,14], at least at ambient pressure. That would make a standard quadrupolar ordering not only at $12 \mathrm{~K}$, but also at $35 \mathrm{~K}$ very unlikely. In order to clarify this question, reliable information on the $\mathrm{CEF}$ excitation energies is crucial.

It is worth noting that such a jumplike drastic increase of the transition temperature under the application of very moderate pressure is highly unusual for Ce-based metals and has no simple explanation in terms of RKKY/Kondo competition, and some authors [9] proposed a metaorbital transition scenario to describe the appearance of a pressureinduced ordered phase. The concept of the metaorbital transition was proposed by Kazumasa Hattori [16]. He investigated a two-orbital Anderson lattice model (orbital energy splitting is induced by the CEF effect) with Ising orbital intersite exchange interactions using a dynamical mean-field theory. It was shown that, if the hybridization between the ground-state $f$-electron orbital and conduction electrons is smaller than the one between the excited $f$-electron orbital and conduction electrons at low pressures, the occupancy of the two orbitals changes steeply upon application of pressure. In other words, the excited CEF excitations, which typically had been ignored, because in most cases the lowest excited CEF state is well separated to the ground state, may start to contribute to the ground state properties and induce the transition. Such a metaorbital transition has been theoretically predicted to happen in $\mathrm{CeCu}_{2} \mathrm{Si}_{2}$ [17], but no experimental verification exists so far in any compound. Therefore, knowledge of the CEF splitting scheme, the magnon excitations, and their pressure evolution can provide crucial information about the unusual physics of $\mathrm{CeCoSi}$, which might be the first realization of a material exhibiting a metaorbital transition.

To address these questions we synthesized polycrystalline samples of CeCoSi and its nonmagnetic counterpart LaCoSi. Then, we characterized the samples using neutron diffraction and specific heat measurements. The magnetic structure and excitation spectra were investigated by means of elastic and inelastic neutron scattering under hydrostatic pressures up to $1.5 \mathrm{GPa}$.

\section{EXPERIMENTAL DETAILS}

The polycrystalline samples of CeCoSi and its nonmagnetic counterpart LaCoSi were synthesized from elemental Ce (La), Co, and Si materials mixed in stoichiometric ratios using arc-melting technique, and then annealed for $\sim 2$ weeks at a temperature close to $1200^{\circ} \mathrm{C}$ (the details are given in [9]). The resulting materials were examined using $\mathrm{x}$-ray powder diffraction and energy dispersive $\mathrm{X}$-ray spectroscopy analysis (EDX). The EDX measurements have shown that, after the annealing, the majority of the sample consists of the CeCoSi phase, with a small inclusion of an elemental $\mathrm{Ce}$ and $\mathrm{CeCo}_{2} \mathrm{Si}_{2}$ phase, but according to powder diffraction, the concentration of impurity phases is below $2 \%$.

Neutron powder diffraction measurements were performed at the diffractometer E6 (HZB facility). The powder diffraction patterns were collected at $T=1.7$ and $20 \mathrm{~K}$ with $\lambda=$ $2.41 \AA$. Inelastic neutron scattering (INS) measurements at ambient pressure were carried out at the time-of-flight (TOF) spectrometers IN4 and IN6 of the Institut Laue-Langevin in the temperature range $1.7-150 \mathrm{~K}$. The incident neutron energies were fixed to $E_{\mathrm{i}}=31.95 \mathrm{meV}$ and $E_{\mathrm{i}}=3.86 \mathrm{meV}$ at IN4 and IN6 experiments, respectively. In these experiments we measured $\sim 10 \mathrm{~g}$ of powder samples.

To study the effect of hydrostatic pressure on the spin excitations in $\mathrm{CeCoSi}$ we performed two INS experiments using the cold TOF spectrometers LET [18] at ISIS neutron source and CNCS $[19,20]$ at SNS, ORNL. In order to apply hydrostatic pressure in both experiments we used similar $\mathrm{NiCrAl}$ pressure cells with a relatively weak background in the inelastic channel and reasonable neutron transmission of $\sim 30 \%$ designed by Dr. R. Sadykov from the Institute for Nuclear Research, Moscow. The cells were filled with $\sim 1.5 \mathrm{~g}$ of powder and fluorinert FC-770 was used as a pressure transmitting medium.

At the CNCS experiment we measured magnetic excitations with two neutron incident energies $E_{\mathrm{i}}=6.15 \mathrm{meV}$ and $25.23 \mathrm{meV}$ to study magnon and CEF excitations, respectively. The measurements were performed at the base temperature of the orange cryostat, $T=1.7 \mathrm{~K}$, and at three pressures of $P=0.2,0.6$, and $1 \mathrm{GPa}$. The pressure cell used in the CNCS experiment had an optical window, which allowed us to monitor the pressure by means of a ruby fluorescence method [21,22].

For our experiment on CeCoSi the LET time-of-flight spectrometer had the special advantage of the so-called multirepetition mode [18], which allows one to perform the measurements with several incident neutron energies at the same pulse. Thereby, we could optimize the incident neutron energies in a way to simultaneously measure magnon and CEF excitations, and therefore decrease the counting time needed for a scan at a given temperature and pressure by a factor of 2 . In our experiment we collected data with three $E_{\mathrm{i}}=3.43,6.8$, and $19 \mathrm{meV}$ in the high flux mode [23]. To further decrease the background scattering we used a small radial collimator with $\mathrm{Gd}_{2} \mathrm{O}_{3}$ painted blades and acceptance diameter of $\sim 4 \mathrm{~mm}$, which was installed directly on the pressure cell inside the cryostat. The data were collected in the temperature range $1.7-100 \mathrm{~K}$. The pressure was calculated from the applied press force taking into account the data from the CNCS experiment.

The recorded data were reduced and analyzed using JANA2006 [24], DAVE [25], MANTID [26], and LAMP [27] software packages. Specific-heat measurements were carried out using a commercial PPMS from Quantum Design at temperature range $1.8-300 \mathrm{~K}$.

\section{EXPERIMENTAL RESULTS}

\section{A. Magnetic structure at ambient pressure}

To characterize the magnetic structure of $\mathrm{CeCoSi}$ we measured neutron powder diffraction using the E6 diffrac- 

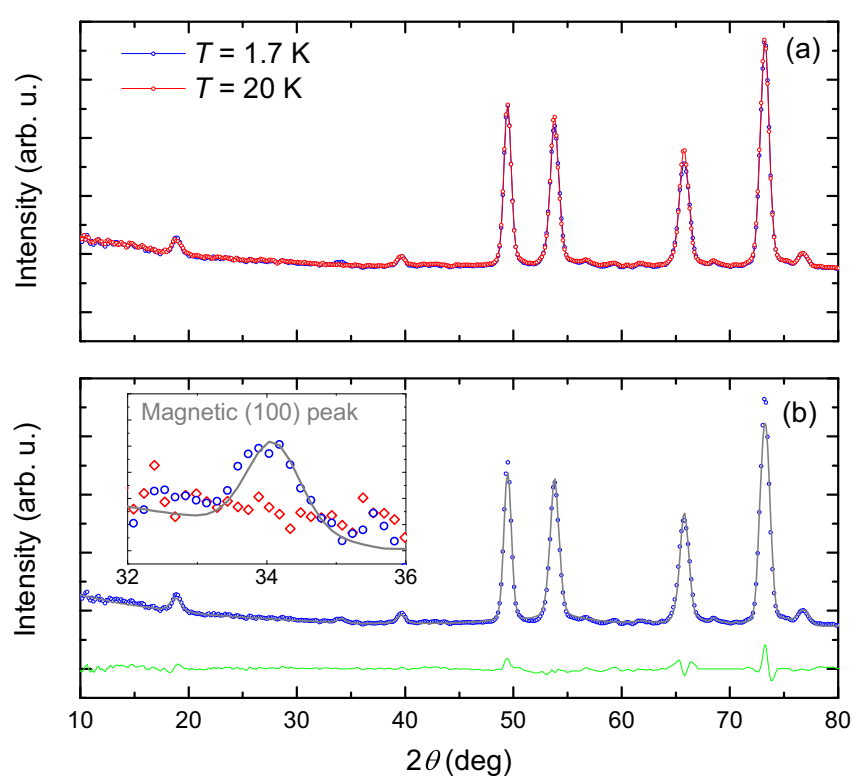

FIG. 1. (a) Neutron powder diffraction of CeCoSi collected at $T=1.7$ and $20 \mathrm{~K}$ at E6 instrument, HZB facility. (b) Refinement of $T=1.7 \mathrm{~K}$ diffraction data [blue points, experimental results; gray line, calculated curve; green, difference; inset shows the zoom of magnetic (100) Bragg peak].

tometer at $\mathrm{HZB}$. The powder diffraction patterns were collected at $T=1.7$ and $20 \mathrm{~K}$, i.e., below and above the $T_{\mathrm{N}}$ and the experimental results are shown in Fig. 1(a). One can see that with decreasing temperature a new weak magnetic satellite appears at $2 \theta \approx 34^{\circ}$ [see inset in Fig. 1(b)]. The peak can be indexed as $\mathbf{k}=(100)$ [note that the (100) nuclear reflection is forbidden for the $P 4 / \mathrm{nmm}$ space group].

We performed magnetic group representation analysis using JANA2006 software and found that the magnetic symmetry group $P m m^{\prime} n$ provides the best fit of our data set. The low-temperature diffraction pattern along with the calculated curve is shown in Fig. 1(b), and one can see a good agreement $\left(R_{\mathrm{nuc}}=2.45 \%\right.$ and $\left.R_{\mathrm{mag}}=4.84 \%\right)$. The lattice parameters of the CeCoSi at $T=1.7 \mathrm{~K}$ were determined to be $a=3.9967(8) \AA$ and $c=6.937(1) \AA$ with the space group $P 4 / n m m$ (values in brackets denote the $1 \sigma$ error of the leastsquares fitting throughout the paper).

The magnetic structure (schematically shown in Fig. 2) turned out to be a collinear antiferromagnetic stacking of ferromagnetic Ce layers along the $c$ axis, with the moments pointing along the [100] direction. The ordered Ce moment is as small as $m_{\mathrm{Ce}}=0.37(6) \mu_{\mathrm{B}}$. It is worth noting that even though our results are consistent with data obtained for the isostructural CeCoGe [13], both analyses are based on a single (100) magnetic reflection, and therefore should be considered with care. Further single-crystal neutron diffraction experiments are highly desirable to confirm the proposed magnetic structure.

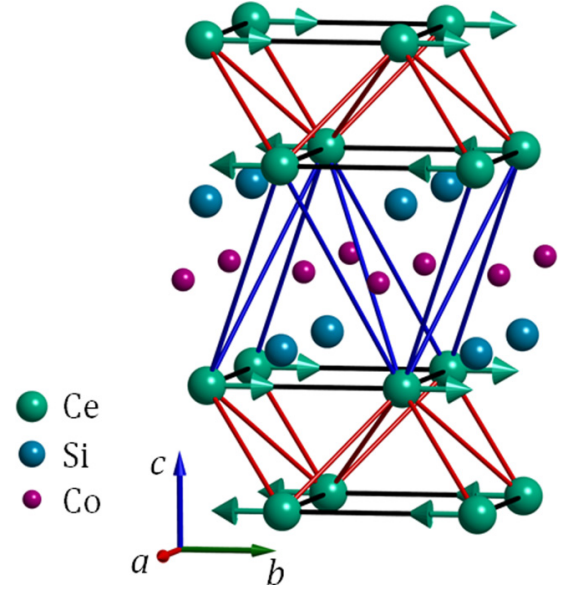

FIG. 2. Sketch of crystal and magnetic structure of CeCoSi. Solid lines show the minimum set of three exchange interactions, which are needed to stabilize the magnetic ground state.

\section{B. Spin excitations at ambient pressure}

\section{Magnon excitations}

To explore the low-energy excitations of CeCoSi we performed powder INS measurements at the spectrometer IN6 at ILL at $T=1.7-100 \mathrm{~K}$. Figure 3 shows the energy spectra collected with $E_{\mathrm{i}}=3.86 \mathrm{meV}$ and integrated within $\mathbf{Q}=$ [1-1.5] $\AA^{-1}$. The low temperature spectrum consists of a strong gapped magnon band at $E \approx 2.5 \mathrm{meV}$. With increasing temperature above $T_{\mathrm{N}}$ the gap closes and the spectral weight transfers to the quasielastic channel as expected for a conventional antiferromagnet.

Note that the characteristic energy of the magnetic excitations in $\mathrm{CeCoSi}$ is $\sim 2.5 \mathrm{meV}$, which is approximately three times higher than the energy associated with the Néel ordering of Ce moments $\left(T_{\mathrm{N}}=8.8 \mathrm{~K} \approx 0.75 \mathrm{meV}\right)$. This may indicate the presence of magnetic frustration or low-dimensional magnetic behavior of the system. Unfortunately, the powder spectrum appears to be almost featureless, which does not allow us to extract specific details of the underlying magnetic interactions. Therefore, the determination of the low-energy spin Hamiltonian, which should contain at least three exchange interactions plus three parameters describing the anisotropy
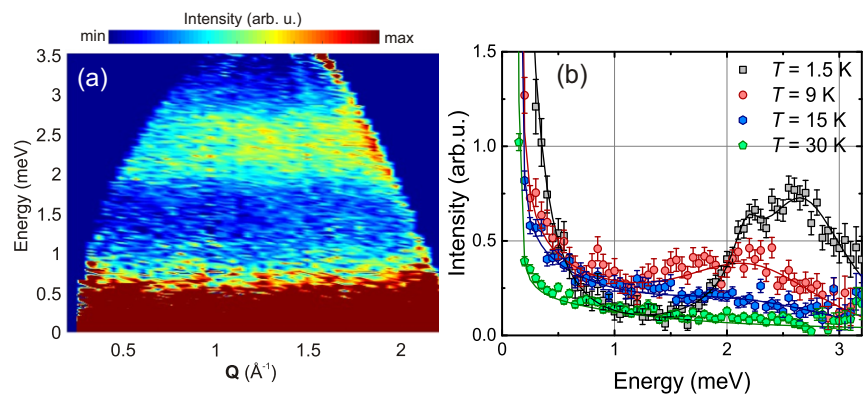

FIG. 3. (a) Low energy INS spectra of CeCoSi measured at IN6 instrument at $T=1.7 \mathrm{~K}$ with $E_{\mathrm{i}}=3.86 \mathrm{meV}$. (b) Temperature dependence of the energy spectra of CeCoSi integrated within $\mathbf{Q}=$ [1-1.5] $\AA^{-1}$. Error bars throughout the text represent one standard deviation ( $1 \sigma$ error). 

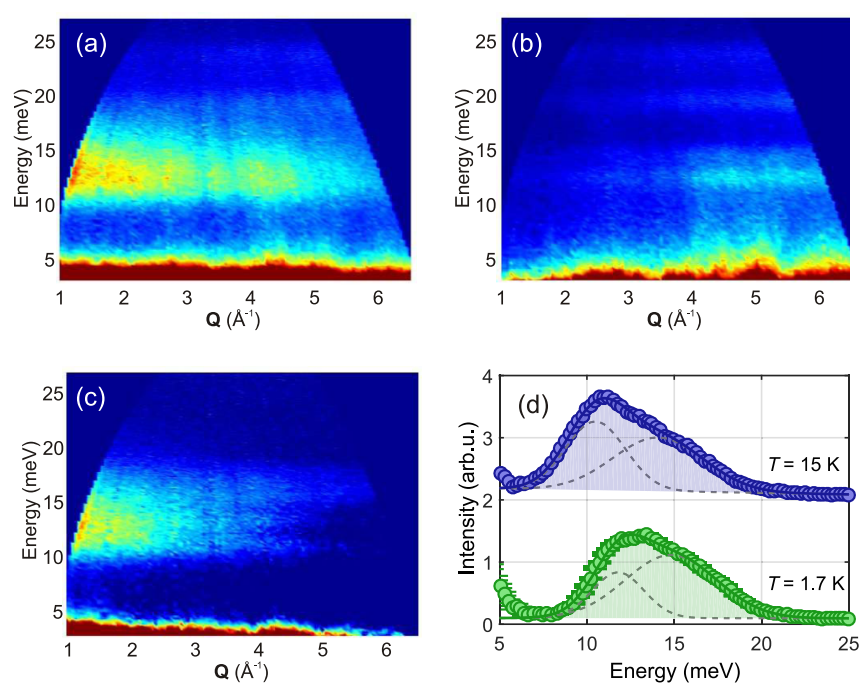

FIG. 4. High-energy INS powder spectra of CeCoSi (a) and $\mathrm{LaCoSi}$ (b) measured at the instrument IN4 at $T=1.7 \mathrm{~K}$ with $E_{\mathrm{i}}=31.95 \mathrm{meV}$. (c) Magnetic signal obtained after subtraction of the scaled LaCoSi spectrum $(\alpha=1.2)$ from the CeCoSi data set. (d) Background subtracted excitation spectra of CeCoSi above and below the $T_{\mathrm{N}}$. Gray dotted lines show the deconvolution of the signal into two Gaussian functions. The data are integrated within $\mathbf{Q}=[1-3] \AA^{-1}$ and are vertically shifted for clarity.

of the exchanges, requires further detailed single-crystal INS measurements.

\section{CEF excitations}

$\mathrm{Ce}^{3+}$ in CeCoSi has a $J=5 / 2$ ground state multiplet, which splits into three doublets under the action of a tetragonal CEF. Thereby, one can expect to observe two CEF transitions in an INS spectrum. To characterize the CEF Hamiltonian in $\mathrm{CeCoSi}$ we performed INS measurements of CeCoSi and $\mathrm{LaCoSi}$ at the TOF instrument IN4 of the Institut LaueLangevin. The spectra of both samples collected at $T=1.7 \mathrm{~K}$ with $E_{\mathrm{i}}=31.95 \mathrm{meV}$ are displayed in Figs. 4(a) and 4(b) [28]. The spectrum of LaCoSi shows strong optical phonon bands, with their intensities increasing with $\mathbf{Q}$ because of the phonon form factor. The spectrum of CeCoSi shows similar phonon bands at large $\mathbf{Q}$, but in addition exhibits broad magnetic excitations at energies $E \approx 10-20 \mathrm{meV}$.

To obtain the magnetic signal of $\mathrm{CeCoSi}$ we directly subtracted the scaled phonon contribution estimated using the LaCoSi data [29]. To find the scaling coefficient $\alpha$, we took an energy cut at high momentum, which is dominated by the phonon contribution in both $\mathrm{La}$ and $\mathrm{Ce}$ samples, because of the phonon and magnetic form factors. To compensate the difference of the sample masses and scattering lengths we scaled the LaCoSi data set to get the best agreement between the spectra. Then, we used the obtained coefficient $\alpha$ to scale the LaCoSi spectrum in the whole $\mathbf{Q}$ range and subtract it from the CeCoSi spectrum $S_{\mathrm{M}}(\mathbf{Q}, \hbar \omega)=S_{\mathrm{Ce}}(\mathbf{Q}, \hbar \omega)-\alpha S_{\mathrm{La}}(\mathbf{Q}, \hbar \omega)$. The magnetic spectrum after subtraction is displayed in Fig. 4(c).

To qualitatively extract the positions of CEF peaks we integrated the magnetic spectrum at $\mathbf{Q}=[1-3] \AA^{-1}$. Two representative curves taken at $T=1.7$ and $15 \mathrm{~K}$ are shown in Fig. 4(d). Note that the error introduced in the energy cuts when not considering the magnetic form factor and the missing data at small $\mathbf{Q}$ for higher energies is well below the symbol size of the data points and similar in size to the statistical error. One can see that the peak shape is rather asymmetric and cannot be fitted with a single peak function and, therefore, to qualitatively extract the peak positions we fitted the curves with two Gaussian peaks. We found that the peaks are located at $E_{1}=10.49(6) \mathrm{meV}$ and $E_{2}=14.1(2) \mathrm{meV}$ at $T=15 \mathrm{~K}$, i.e., above $T_{\mathrm{N}}$, and their positions slightly shift in the antiferromagnetic phase at $T=1.7 \mathrm{~K}\left[E_{1}=11.78(6) \mathrm{meV}\right.$ and $\left.E_{2}=14.8(3) \mathrm{meV}\right]$ due to the splitting of the ground state doublet by an exchange field. It is worth noting that the CEF excitations are broader than the instrumental resolution, which may be due to the interaction with phonons [30], hybridization with the conduction band electrons, or magnetic dispersion.

\section{Specific heat}

To check whether the broad asymmetric peak observed in the INS spectra indeed consists of two CEF excitations we carefully measured the heat capacity of the $\mathrm{CeCoSi}$ and LaCoSi samples over a wide temperature range $T=1.8-300 \mathrm{~K}$ using a PPMS. Specific heat of the LaCoSi sample was used as a blank to estimate the phonon contribution and calculate the magnetic contribution in $\mathrm{CeCoSi}$.

The raw data and the magnetic heat capacity $C_{\text {mag }}$ after subtraction of the phonon contribution are shown in Fig. 5. $C_{\text {mag }}(T)$ exhibits two anomalies: a sharp peak at $T_{\mathrm{N}}$ and a broad Schottky-like anomaly with a maximum at $T^{*}=$ $51.5 \mathrm{~K}$.

First of all, we focus on the high-temperature part of the specific heat curve. One can see that the absolute value of the specific heat $C\left(T^{*}\right)=5.7 \mathrm{~J} / \mathrm{mol} \mathrm{K}$ significantly exceeds the $3.65 \mathrm{~J} / \mathrm{mol} \mathrm{K}$ expected for a simple Schottky anomaly for a doublet-doublet transition. In contrast, the $C\left(T^{*}\right)$ is only slightly lower than $6.31 \mathrm{~J} / \mathrm{mol} \mathrm{K}$-the peak specific heat expected for a doublet-quartet transition. This indicates that the anomaly is caused by two close standing CEF transitions. Also, from the $T^{*}$ we can estimate the energy gap between the doublet and excited quasiquartet states $\Delta \approx 11.8 \mathrm{meV}$. Note that this result is in a very good agreement with the mean energy of two doublets observed in our INS measurements $\left(E_{1}+E_{2}\right) / 2=12.3 \mathrm{meV}$. To qualitatively calculate the hightemperature magnetic specific heat of $\mathrm{CeCoSi}$ we used the standard equation for the specific heat of a discrete $n$-level system:

$$
C(T)=N_{\mathrm{Av}} k_{\mathrm{B}} \frac{\delta}{\delta T}\left(\frac{1}{\mathcal{Z}} \sum_{i=1}^{n} E_{\mathrm{i}} e^{-\frac{E_{\mathrm{i}}}{k_{\mathrm{B}} T}}\right),
$$

where $E_{\mathrm{i}}$ are energies of states and $\mathcal{Z}$ is a partition function. Using Eq. (1) and transition energies $E_{1}=10.49(6) \mathrm{meV}$ and $E_{2}=14.09(21) \mathrm{meV}$ determined by INS above $T_{\mathrm{N}}$ we calculated the magnetic specific heat of $\mathrm{CeCoSi}$, and the results are plotted in Fig. 5(b) by the red line. The good agreement between calculated and measured specific heat curves provides another evidence that the CEF transition energies determined by INS are valid. The deviation between the measured and 

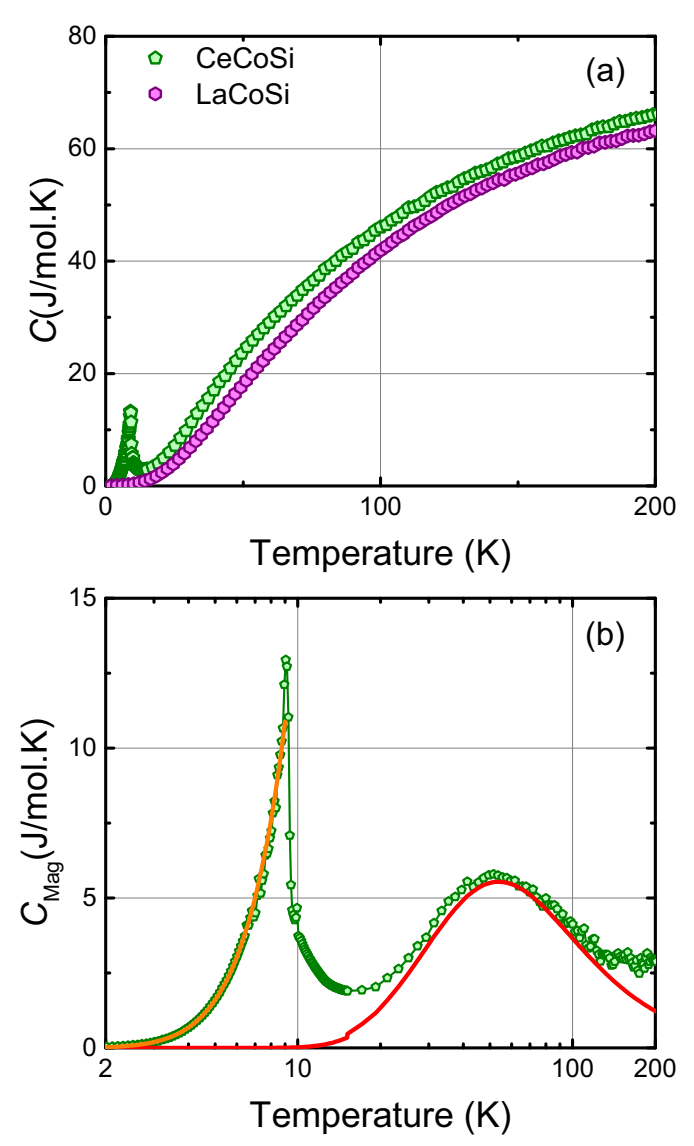

FIG. 5. (a) Temperature dependences of CeCoSi and LaCoSi specific heat $C(T)$. (b) Magnetic part $C_{\text {mag }}$ of the specific heat of CeCoSi versus temperature $T$ in a semilogarithmic plot. The solid red and orange lines show the fits of CEF and magnon contributions to the specific heat using Eq. (1) and Eq. (2), respectively.

the calculated specific heat curves at high temperature above $\sim 130 \mathrm{~K}$ are caused by the inaccuracy due to subtraction of a massive phononic contribution, which dominates at high temperature.

The low-temperature part of the specific heat contains information about the magnon density of state due to the magnetic ordering. For instance, the specific heat of the 3D Heisenberg AFM follows a simple power law $C \propto T^{3}$ due to the 3D gapless dispersion with $\hbar \omega \propto \mathbf{k}$. On the other hand, if the system has a magnon gap one would expect an activation behavior $C \propto e^{-\Delta / k_{\mathrm{B}} T}$. For the gapped magnons in a threedimensional magnetic metal the low-temperature part of the specific heat can be expressed as [31]

$C(T)=\gamma T+b \Delta^{\frac{7}{2}} T^{\frac{1}{2}} e^{-\Delta / k_{\mathrm{B}} T}\left[1+\frac{39}{20}\left(\frac{T}{\Delta}\right)+\frac{51}{32}\left(\frac{T}{\Delta}\right)^{2}\right]$.

The first term $\gamma T$ describes the electronic contribution to the specific heat; $b$ is the constant inversely proportional to the spin-wave velocity $b \propto\left(\frac{1}{D}\right)^{3}$.

We fitted the low- $T$ part of our specific heat curve $(2<$ $T<\frac{2}{3} T_{\mathrm{N}} \approx 6 \mathrm{~K}$ ) using Eq. (2). The fitted curve is shown in Fig. 5(b) by orange line and one can see the perfect agreement between the experimental and calculated curves. It is interesting to note that the extrapolation of our fit function to higher temperature up to $8 \mathrm{~K}$ provides surprisingly good description of the observed specific heat data.

The fitted parameters were found to be $\gamma=23.9(6)$ $\mathrm{mJ} / \mathrm{mol} \mathrm{K}^{2}$ and $\Delta / k_{\mathrm{B}}=12.75(7) \mathrm{K}$. It is worth noting that the gap determined from the specific heat measurements is of the order of the ordering temperature of $\mathrm{CeCoSi}$.

\section{Magnetic excitations under hydrostatic pressure}

We start our presentation of the pressure-induced evolution of the spin dynamics in $\mathrm{CeCoSi}$ with the spectra collected at the LET spectrometer. Note that the pressure cell produces a massive background signal. In order to determine the nonmagnetic scattering we used the LaCoSi spectrum measured under similar conditions and the procedure described in Sec. III B 2 assuming that $S_{\mathrm{M}}(\mathbf{Q}, \hbar \omega)=S_{\mathrm{Ce}}(\mathbf{Q}, \hbar \omega)-$ $\alpha \cdot S_{\mathrm{La}}(\mathbf{Q}, \hbar \omega)$. However, even without the subtraction a strong broad excitation band at $E \approx 4 \mathrm{meV}$ is clearly seen in the spectrum [the raw spectra obtained on the LET spectrometer are presented in Appendix A, Figs. 11(a)-11(d)].

As was discussed above, in this experiment we did not have a pressure sensor in the cell, and the pressure of $1.5 \mathrm{GPa}$ was calculated from the applied press force taking into account $\sim 10 \%$ loss, while cooling down to $1.7 \mathrm{~K}$, which results in the relatively large estimated uncertainty of the pressure determination of $\sim 0.25 \mathrm{GPa}$. For this reason we decided to study the $T$ dependence of the observed mode at fixed $P$. We subtracted the background and Bose-corrected all obtained spectra measured with $E_{\mathrm{i}}=6.8 \mathrm{meV}$. The resulting $\chi^{\prime \prime}(\hbar \omega)$ curves integrated within $\mathbf{Q}=[0.5-2.5] \AA^{-1}$ are shown in Fig. 6(a). Increasing temperature induces a decrease of the mode intensity, and slightly shifts down the peak position. Fits of these parameters are presented in Figs. 6(b) and 6(c) and one can see that the magnon mode intensity disappears below the detection limit at $T=30 \mathrm{~K}$. This result is in a reasonable agreement with the phase diagram of $\mathrm{CeCoSi}$, which shows transition temperature of $\sim 35 \mathrm{~K}$ at $P \approx 1.5 \mathrm{GPa}$.

In order to check the consistency of our results with the zero pressure data we also measured the spectra at almost ambient condition $(P \leqslant 0.1 \mathrm{GPa})$ at $1.7 \mathrm{~K}$. The resulting spectrum along with the $1.5 \mathrm{GPa}$ data and results of the IN4 experiment are shown in Fig. 7. The position of the CEF excitations obtained in the LET experiment perfectly coincides with the IN4 results indicating that we can reliably extract information about both CEF and magnon excitations from the LET data. It is interesting to note that the pressure of $P=1.5 \mathrm{GPa}$ significantly shifts or suppresses the intensity of the CEF excitations as clearly seen in Fig. 7.

We now focus on the pressure dependence in more detail and present data obtained on the CNCS spectrometer. Figure 8 shows the summary of the background subtracted signal at $0.2,0.6$, and $1.0 \mathrm{GPa}$ collected with two incident neutron energies [32]. The $E_{\mathrm{i}}=6.15 \mathrm{meV}$ spectra presented in the left panel display a rather strong magnon peak, the position of which gradually shifts upon increasing pressure. It is worth noting that already at the lowest pressure of $0.2 \mathrm{GPa}$ the position of the peak is slightly higher than the one obtained in our IN6 experiment at ambient pressure. 

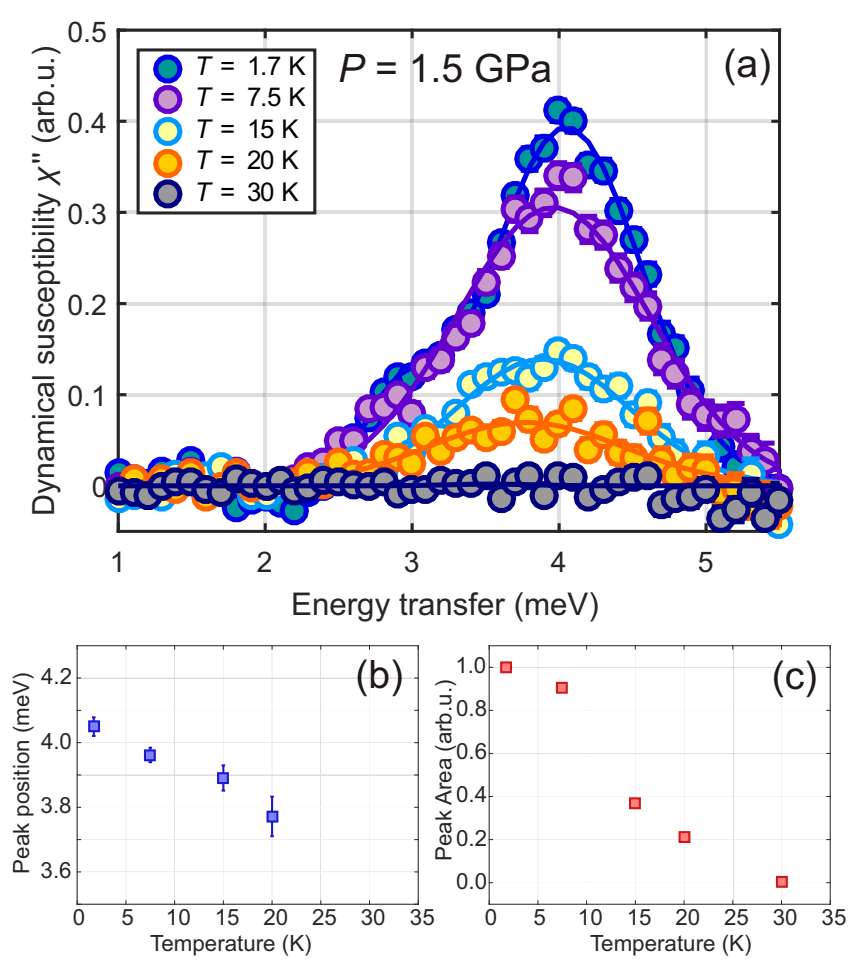

FIG. 6. Temperature dependence of INS signal at $P=1.5 \mathrm{GPa}$. (a) INS spectra taken at $P=1.5 \mathrm{GPa}$ with $E_{\mathrm{i}}=6.8 \mathrm{meV}$ and integrated within $\mathbf{Q}=[0.5-2.5] \AA^{-1}$. (b), (c) Magnon peak position and integrated intensity as functions of temperature.

The high-energy data have much stronger background due to the phonon scattering from the pressure cell. The data after subtracting the background contribution are shown in panel (b) of Fig. 8. At $P=0.2 \mathrm{GPa}$ we found a weak peak at an energy of $\sim 13 \mathrm{meV}$. Its position is close to $E_{1}=$ $11.78(6) \mathrm{meV}$ and $E_{2}=14.8(3) \mathrm{meV}$ observed in the IN4 experiment at ambient pressure. The position of the peak also shifts to higher energies with pressure. However, the signal-tonoise ratio is much worse in the $25.23 \mathrm{meV}$ data set compared to the $6.15 \mathrm{meV}$ one, as can be seen from the ratio between the

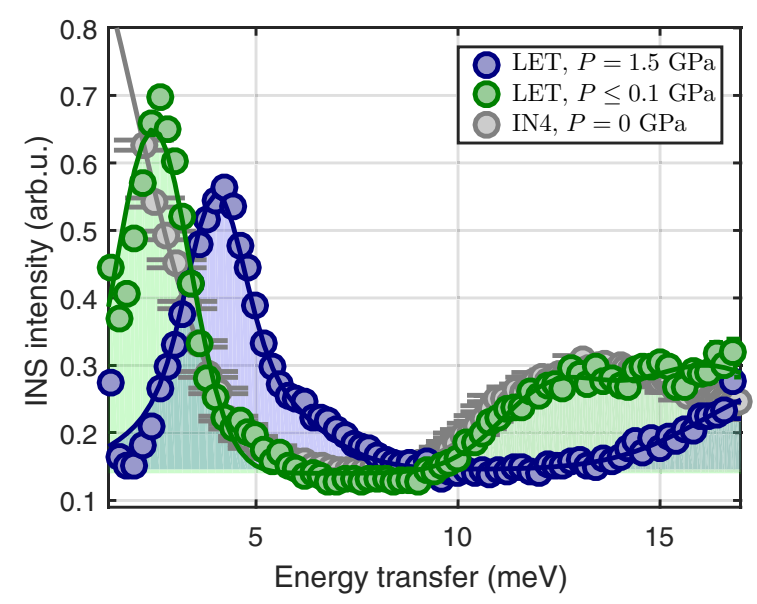

FIG. 7. Background subtracted INS spectra of CeCoSi taken at $T=1.7 \mathrm{~K}$ on LET and IN4 instruments. The data are integrated within $\mathbf{Q}=[0.5-2.5] \AA^{-1}$.
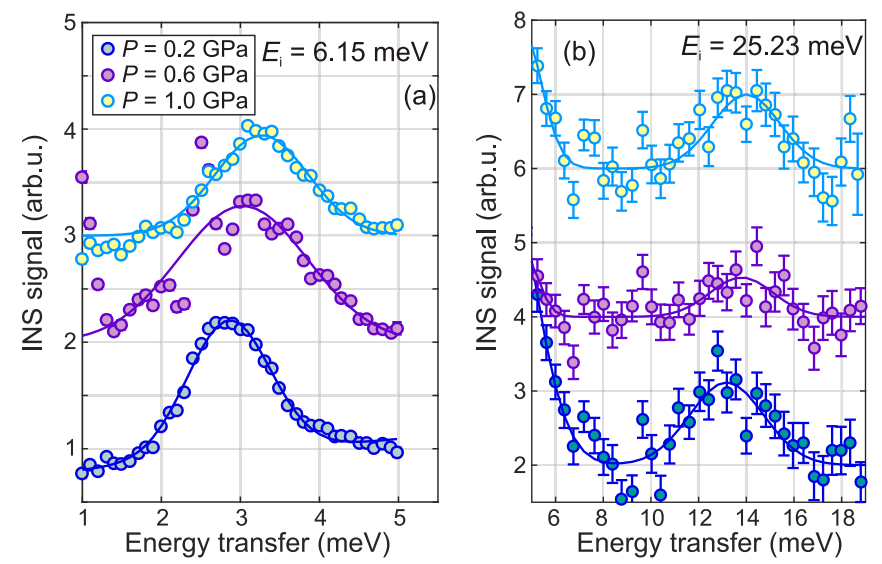

FIG. 8. Magnetic signal of CeCoSi after background subtraction measured using CNCS at $T=1.7 \mathrm{~K}$ with $E_{\mathrm{i}}=6.15 \mathrm{meV}$ (a) and $E_{\mathrm{i}}=25.23 \mathrm{meV}(\mathrm{b})$. The data are integrated within $\mathbf{Q}=[1-2.5] \AA^{-1}$ and $\mathbf{Q}=[1.8-3] \AA^{-1}$ in left and right panels, respectively. Different data sets are shifted vertically for ease of viewing.

neutron count rate and error bars in the two panels of Fig. 8, and the $13 \mathrm{meV}$ peak has an intensity which exceeds the background level by $2-4$ standard deviations only. Taking into account that the positions and intensities of the peaks would depend on the details of the subtraction procedure, we would like to point out that the obtained result should be considered with a reasonable caution, because we cannot unambiguously prove a magnetic origin of the observed peak, which can be just an artifact of the background subtraction procedure [33].

On the other hand, the fitting of the low-energy peak [Fig. 8(a)] is rather robust and self-consistent, independent on subtraction details. Accordingly, we can conclude that the energy of the magnon mode indeed increases with the pressure, whereas the observation of the CEF excitations and their $P$ dependence is much more questionable.

\section{DISCUSSION}

Our experimental work on CeCoSi has a dual aim: (i) to characterize the magnetic ground state and excitations of $\mathrm{CeCoSi}$ at ambient pressure using a combination of different techniques and (ii) to study how the magnetic excitations evolve with pressure. Analyzing the results of neutron powder diffraction in the AFM phase and at ambient pressure we detected only one weak magnetic satellite peak, which appears below $T_{\mathrm{N}}$ and can be indexed as the (100) reflection. This result is consistent with a simple commensurate antiferromagnetic structure, previously proposed for isostructural $\mathrm{CeCoGe}$ [13]. The Ce moments are aligned along the [100] direction and carry an ordered moment of only $m_{\mathrm{Ce}}=0.37(6) \mu_{\mathrm{B}}$, which is significantly reduced compared to the moment of free $\mathrm{Ce}^{3+}$ with $m_{\mathrm{Ce}}=2.14 \mu_{\mathrm{B}}$. However, in Ce systems the CEF is comparatively strong and therefore the $J=5 / 2$ multiplet is split in such a way that the energy of the first excited CEF level is in general much larger than $T_{\mathrm{N}}$. Then, the size of the ordered moment is limited to that of the CEF ground state doublet, which for the easy CEF direction is in the range $1-2.5 \mu_{\mathrm{B}}$ [34]. But even compared to the lower bound, the observed value is small. This is striking, because the $4 f$ entropy 
collected just above $T_{\mathrm{N}}$ is close to $R \ln 2$. This indicates that only a very small amount of the $4 f$ entropy connected with the ground state CEF doublet is collected above $T_{\mathrm{N}}$. Since the onset of correlations is always associated with the reduction of entropy, the amount of correlation above $T_{\mathrm{N}}$ within the CEF ground state doublet cannot be large, and therefore these correlations should not be able to result in a strong reduction of the ordered moment far below $T_{\mathrm{N}}$. Thus the standard scenarios invoked to account for a reduced size of ordered moments, the presence of Kondo interaction or frustration, do not apply since in these scenarios the reduction of the moment in the ordered state far below $T_{\mathrm{N}}$ is connected with a reduction of the entropy collected at $T_{\mathrm{N}}[35,36]$. Another alternative, which is presently discussed for a number of ferromagnetic systems, the ordering along the hard CEF direction, where the available CEF moment can be very small $[37,38]$, seems to be unlikely because susceptibility data do not indicate a strong anisotropy [14]. Thus the origin of the strong reduction of the ordered moment is a further mystery in this system.

Both the inelastic neutron scattering and the specific heat results demonstrate that the first excited and the second excited CEF doublets are close by in energy and at a mean energy of the order of $12 \mathrm{meV}$ (about $140 \mathrm{~K}$ ). This corroborates the doubts on the possibility of a quadrupolar transition in the temperature range 10-40 K expressed in the Introduction. Because $\mathrm{Ce}^{3+}$ is a Kramers ion and thus each CEF doublet does not bear a quadrupolar degree of freedom, a quadrupolar transition has to be an induced one, a process which is well known for magnetic order in singlet systems. However, that requires the ordering temperature to be larger than typically $\Delta / 2$, where $\Delta$ is the energy splitting between the involved $\mathrm{CEF}$ ground state and excited state. Thus in the present case $T_{\mathrm{Q}}$ should be larger than about $70 \mathrm{~K}$. According to established results for the magnetic singlet-singlet case, in such induced ordering processes the ratio between the ordering temperature $T_{\mathrm{c}}$ and $\Delta$ is given by $T_{\mathrm{c}} / \Delta=\operatorname{arctanh}(\Delta / J)$, where $J$ is a coupling parameter [39]. The arctanh function results in an extremely fast, almost vertical drop of $T_{\mathrm{c}}$ with decreasing $J$ for $T_{\mathrm{c}} / \Delta<0.5$, making the realization and stabilization of such a low $T_{\mathrm{c}} / \Delta<0.5$ very difficult and very unlikely. This almost vertical dependence of $T_{\mathrm{c}} / \Delta$ is, e.g., incompatible with the very smooth and almost linear increase of the proposed quadrupolar transition in $\mathrm{CeCoSi}$ under pressure shown in [10]. This analysis confirms that the transition at $T=12 \mathrm{~K}$ at $P=0$ [14] and at $T \approx 36 \mathrm{~K}$ at $P=1.5 \mathrm{GPa}$ are connected with an unconventional order. However, it is worth noting that in contrast to the results of [14] we did not observe any indication of phase transition at $12 \mathrm{~K}$ at ambient pressure in our data.

In the low-energy spectrum we observed magnon excitations with a characteristic energy of $E^{*} \approx 2.5 \mathrm{meV}(29 \mathrm{~K})$ at ambient pressure. It is worth noting that the excitation energy scale of the magnons exceeds by more than three times the ordering temperature of $T_{\mathrm{N}}=8.8 \mathrm{~K}$. One possible explanation is a quasi-2D magnetic structure of the material (see Fig. 2) with much stronger exchange interactions within the $a b$ planes and only weak coupling along the $c$ direction $\left(J_{\mathrm{c}}<J_{\mathrm{ab}}\right)$. In that case, short-range fluctuations within the $a b$ plane will survive at temperatures above the $T_{\mathrm{N}}$. Indeed, we were able to resolve a broad paramagnon inelastic peak at 9 and $10 \mathrm{~K}$,

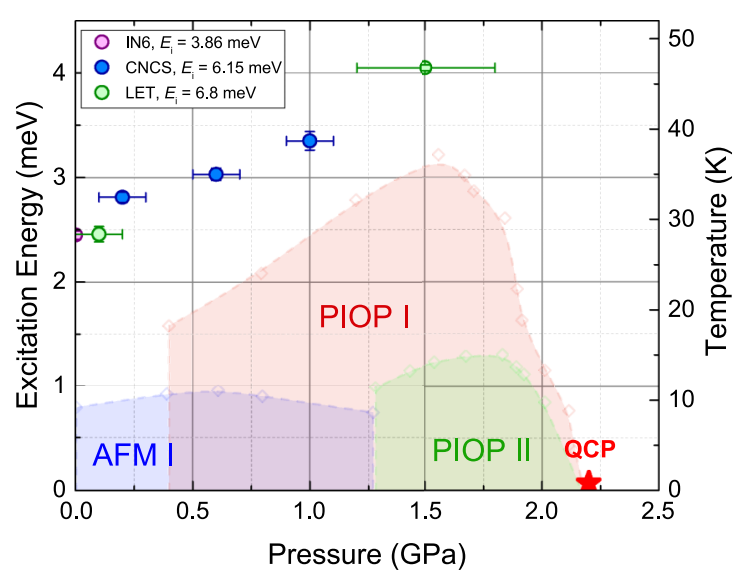

FIG. 9. Temperature-pressure phase diagram of CeCoSi from Ref. [9] and the $P$ dependence of the magnon mode (solid circles) at $T=1.7 \mathrm{~K}$. Shaded areas represent different phases-AFM and two pressure-induced ordered phases (PIOP). Note that the right and left axes are shown on the same scale $E=k_{\mathrm{B}} T$.

inline with such a scenario, whereas at higher temperatures all spectral weight is transferred to the quasielastic channel.

However, the properties of the CEF ground state deduced from our analysis raise a further problem (see Appendix B). The wave function of this CEF ground state corresponds to a $c$-axis moment of $m_{c}=0.97 \mu_{\mathrm{B}}$ and a basal plane moment of $m_{a}=0.56 \mu_{\mathrm{B}}$; thus it is not very anisotropic. This weak anisotropy of the local moment cannot account for the large gap in the magnetic excitation spectrum of the ordered state deduced from the INS and specific heat results. Furthermore, the very weak anisotropy observed in the magnetic susceptibility indicates that the anisotropy of the magnetic interactions is also weak. Thus this large gap in the magnetic excitations is a further open problem in this unusual system.

Summarizing the results of the low-energy INS experiments under pressure, we found that the energy of the magnon mode gradually evolves from $2.5 \mathrm{meV}$ at ambient pressure to $\sim 4 \mathrm{meV}$ at $1.5 \mathrm{GPa}$ (see Fig. 9). At this pressure the energy scale of the magnetic excitations is comparable to the transition temperature of the pressure-induced phase $(4 \mathrm{meV}$ $\approx 46 \mathrm{~K}$ ). Note that these results are not in favor of the metaorbital transition scenario, because the last implies a sharp, abrupt change of the ordered moment and the magnon excitation energy as a consequence, which is in contrast to the gradual pressure-induced evolution observed in our measurements.

The pressure dependence of the CEF excitations is less clear: in the low-pressure $(0.2 \mathrm{GPa}) \mathrm{CNCS}$ experiment we found a weak peak, close to the positions of the CEF excitations observed at zero-pressure measurements. The peak position changes only slightly with pressures up to $1 \mathrm{GPa}$. On the other hand, the results of the LET experiment unambiguously showed that at $1.5 \mathrm{GPa}$ the $\mathrm{CEF}$ levels move out of their original location. One possible explanation is much worse signal-to-noise ratio in the high-energy CNCS measurements, which cast some doubts on the CNCS results. However, if one looks at the signature in the resistivity, the high- $T$ ordering is something new which appears quite abruptly at $P \geqslant 1.4 \mathrm{GPa}$, while the observed effects at lower pressure were different and 
an order of magnitude weaker. Therefore, $P_{1}=1.4 \mathrm{GPa}$ was explicitly introduced to highlight this strong change in [9]. Thus the appearance of the strong anomaly in $\rho(T)$ at $P \geqslant P_{1}$ may be related to the dramatic change of CEF excitations between 1 and $1.5 \mathrm{GPa}$, indicating that there is a real strong difference between the orderings above and below the $P_{1}$, as was suggested in [9]. A single crystal neutron diffraction under pressure should be performed to resolve this question and clarify the order parameter of the PIOP.

\section{CONCLUSION}

To summarize, we performed a comprehensive experimental investigation of $\mathrm{CeCoSi}$ by means of neutron scattering and specific heat measurements. At ambient pressure $\mathrm{Ce}-$ $\mathrm{CoSi}$ orders into a simple AFM structure with a surprisingly small ordered moment of only $m_{\mathrm{Ce}}=0.37(6) \mu_{\mathrm{B}}$ and exhibits spin excitations on two different energy scales: low-energy collective magnons at $\sim 2.5 \mathrm{meV}$ and two CEF transitions at $\sim 12 \mathrm{meV}$. The application of hydrostatic pressure up to $1.5 \mathrm{GPa}$ causes a gradual shift of the magnon band towards higher energies and significantly modifies the CEF splitting scheme at $1.5 \mathrm{GPa}$. The obtained results are not in favor of the metaorbital scenario [16], which was proposed to describe the origin of the pressure-induced phases in $\mathrm{CeCoSi}$ [9].

\section{ACKNOWLEDGMENTS}

We acknowledge A. S. Sukhanov for stimulating discussions, C. Goodway for help with the high-pressure experiments at ISIS Neutron and Muon source, and K. A. Nikitina for assistance with the CEF analysis. This research used resources at the Spallation Neutron Source, a DOE Office of Science User Facility operated by Oak Ridge National Laboratory. S.E.N. acknowledges support from the International Max Planck Research School for Chemistry and Physics of Quantum Materials (IMPRS-CPQM). D.G.F. and C.G. acknowledge support from the German Research Foundation (DFG) through Grant No. GE 602/4-1 and Fermi-NEst.

\section{APPENDIX A: RAW INS DATA}

Figures 10 and 11 show the raw $S(\mathbf{Q}, \omega)$ of $\mathrm{CeCoSi}$ and LaCoSi samples and the magnetic spectra $S_{\mathrm{M}}(\mathbf{Q}, \omega)$ after phonon/background subtraction measured on CNCS and LET spectrometers, respectively.

\section{APPENDIX B: ANALYSIS OF CEF HAMILTONIAN}

The Ce ions in CeCoSi occupy a position with $4 \mathrm{~mm}$ point symmetry, and thereby the crystalline electric field Hamiltonian of the $\mathrm{Ce}^{3+}$ ion will include only three $B_{l}^{m}$ coefficients. In Stevens notation it can be written as

$$
\mathcal{H}=B_{2}^{0} O_{2}^{0}+B_{4}^{0} O_{4}^{0}+B_{4}^{4} O_{4}^{4} .
$$

In the paramagnetic phase the Hamiltonian (B1) exhibits three Kramers doublets; therefore, at low temperatures $\Delta_{\mathrm{CEF}} \gg k_{\mathrm{B}} T$ the INS spectrum consists of two transitions. In Sec. III B 2 we report the observation of two CEF levels at $T=15 \mathrm{~K}$ with energies $\Delta_{1}=10.49(6) \mathrm{meV}$ and $\Delta_{2}=$
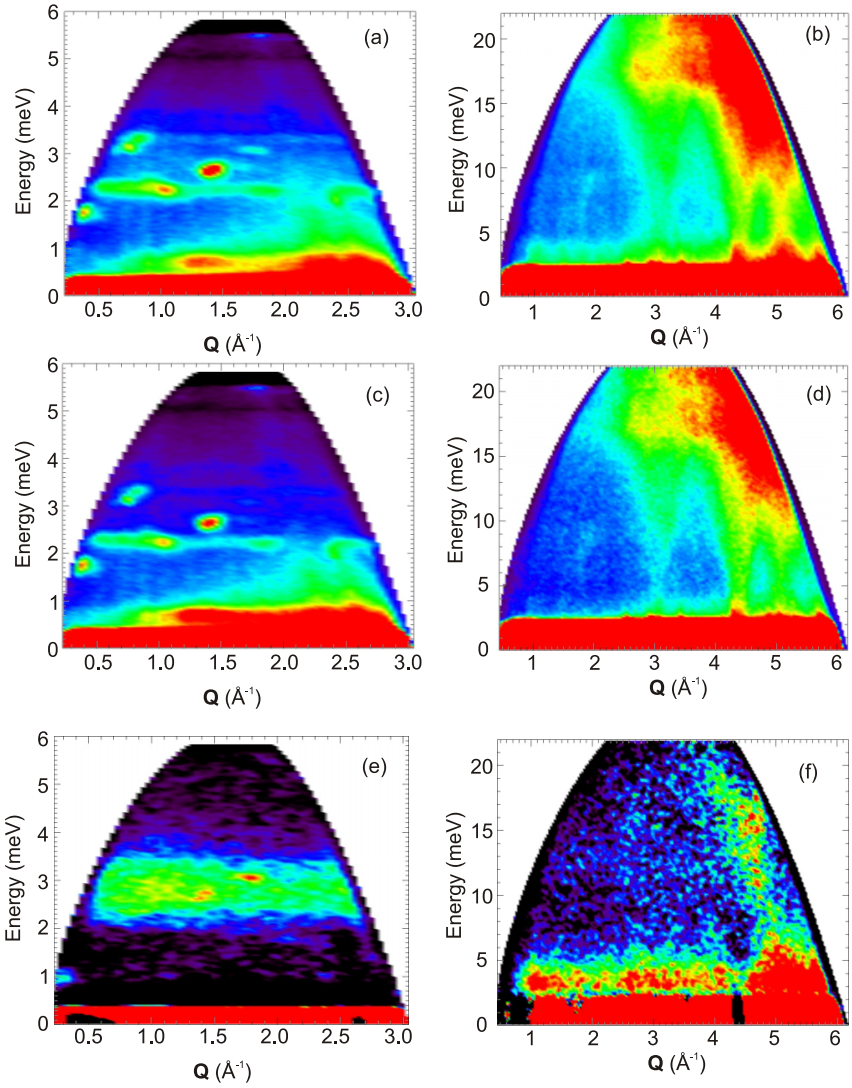

FIG. 10. INS spectra of CeCoSi (a), (b) and LaCoSi (c), (d) measured at $T=1.7 \mathrm{~K}$ on the CNCS spectrometer with $E_{\mathrm{i}}=6.15 \mathrm{meV}$ (left) and $E_{\mathrm{i}}=25.23 \mathrm{meV}$ (right) using a NiCrAl pressure cell at a pressure $P=0.2 \mathrm{GPa}$. (e), (f) INS spectra of CeCoSi after the subtraction of the nonmagnetic LaCoSi contribution. The intensities were scaled by $\times 5$ with respect to the raw spectra (a)-(d) to highlight the observed excitations.

14.1(2) meV. The relative intensity ratio is $I_{1} / I_{2} \approx 0.83$. Taking into account these results along with the temperature dependence of the magnetic susceptibility reported on a single crystalline sample [14] we performed a fitting of the Hamiltonian (B1).

As the first step we fitted the transition energies. For that we defined the deviation as

$$
\chi=\left(\sum_{i}^{2}\left(E_{i}^{\mathrm{calc}}-E_{i}^{\mathrm{obs}}\right)^{2}\right)^{\frac{1}{2}},
$$

and made a "brute-force" search of the $B_{l}^{m}$ coefficients within the parameter space of $B_{2}^{0}=[-2,2] \mathrm{meV}, B_{4}^{0}=[-1,1] \mathrm{meV}$, and $B_{4}^{4}=[-1,1] \mathrm{meV}$ with a step size of $2 \mu \mathrm{eV}$.

To find the $E^{\mathrm{Obs}}$ we calculated the eigenvalues of the Hamiltonian (B1) with the given set of $B_{l}^{m}$. Figure 12(a) shows the sets of parameters which satisfy the condition of $\chi<0.2 \mathrm{meV}$ in three dimensional $B_{l}^{m}$ space.

One can see that the solutions form three rings. We applied density-based spatial clustering DBSCAN as implemented in sklearn library [40] to separate them. Then, we described each of the obtained rings using a singular value decomposition approach (SVD). Simply put, for each ring we shifted the 

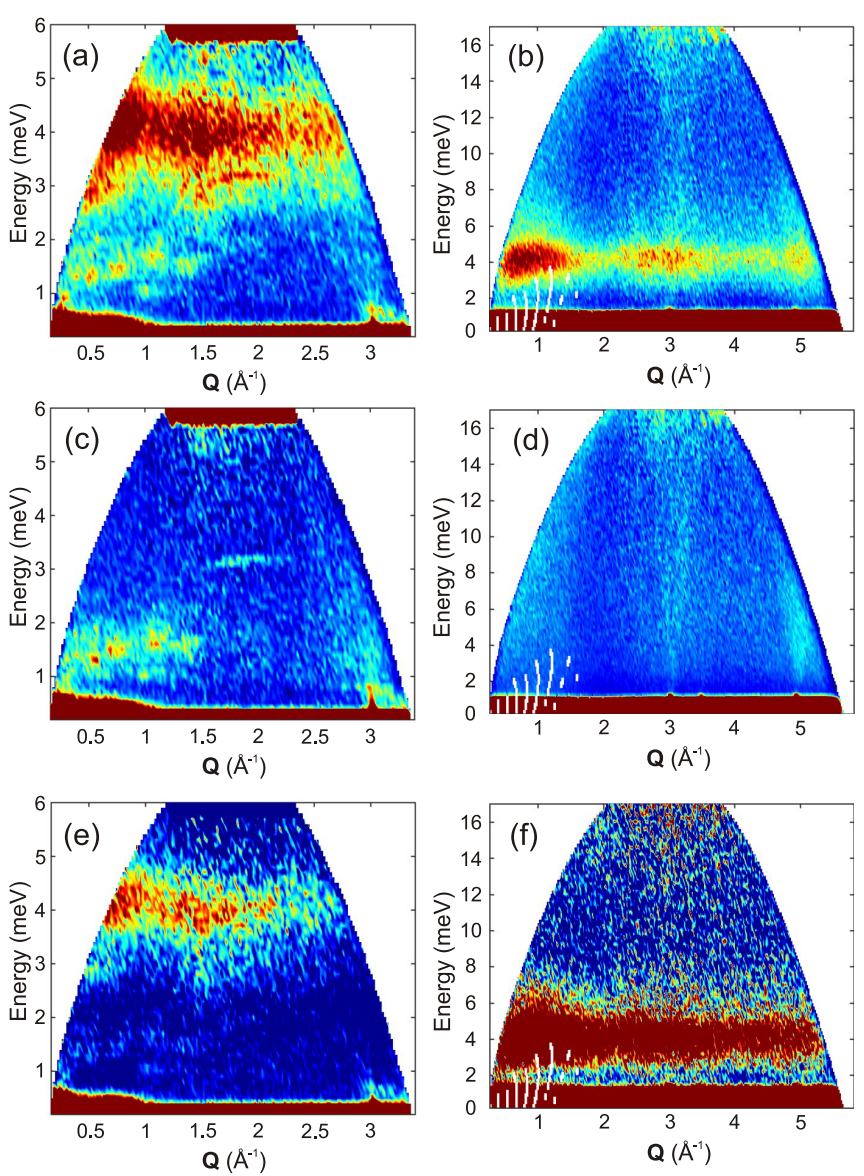

FIG. 11. INS spectra of CeCoSi (a), (b) and $\mathrm{LaCoSi}$ (c), (d) measured at $T=1.7 \mathrm{~K}$ on the LET spectrometer with $E_{\mathrm{i}}=6.8 \mathrm{meV}$ (left) and $E_{\mathrm{i}}=19 \mathrm{meV}$ (right) using a $\mathrm{NiCrAl}$ pressure cell at a pressure $P=1.5 \mathrm{GPa}$. (e), (f) INS spectra of CeCoSi after the subtraction of the nonmagnetic LaCoSi contribution. The intensity of the (f) panel was scaled by $\times 5$ with respect to (b), (d) panels to highlight the excitations.

center of the coordinate system to the mean value: $\hat{B}_{l}^{m} \rightarrow$ $B_{l}^{m}+\overline{B_{l}^{m}}$. After that, we searched for a matrix $M$, which would rotate/deform the coordinate system in a way to approximate the data set by a unit circle. Thus we presume that the solutions have an elliptical shape, which seems to be valid with the experimental precision of the energy determination. Figure 12(a) shows the eigenvalues calculated by this bruteforce method and the fitting with SVD. One can see the excellent agreement between the curves. The obtained values for $\overline{B_{l}^{m}}$ and the rotation matrices $M$ for each solution ring are given in Table I and can be used to recalculate each solution ring.

As the next step we calculated the ratio of the transition intensities $I_{1} / I_{2}$ for the obtained rings using the standard equation for the INS transition intensity:

$$
I\left(\psi_{i} \rightarrow \psi_{j}\right) \propto \sum_{\alpha=x, y, z}\left|\left\langle\psi_{j}\left|J_{\alpha}\right| \psi_{i}\right\rangle\right|^{2} .
$$

Figure 12(c) shows $I_{1} / I_{2}$ for all rings as a function of the angle $\varphi$ from the $B_{4}^{4}$ axis; for definition of $\varphi$, see Fig. 12(b). Based on $I_{1} / I_{2}=0.83(6)$ as determined in the experiment we con-
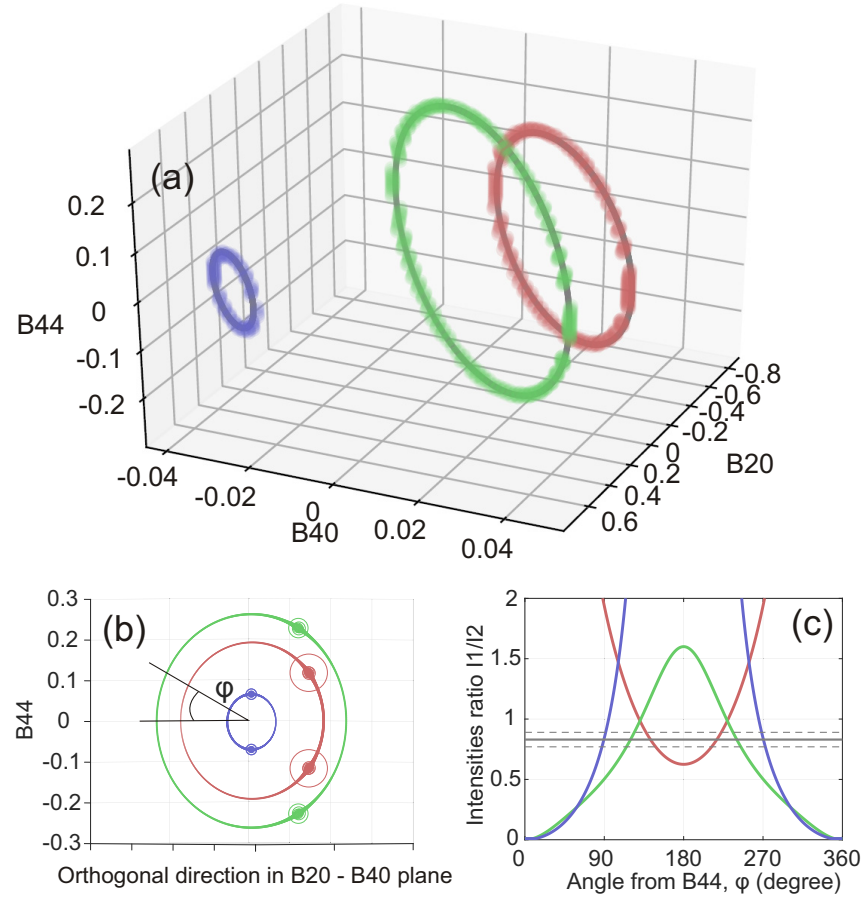

FIG. 12. Fitting of the CEF Hamiltonian. (a) Semitransparent color rings show the parameter space of the Hamiltonian (B1) with low cost function Eq. (B2) $\chi<0.2 \mathrm{meV}$. The three colors are used to indicate different parameter regions. The gray solid lines show the individual fitting of those regions using SVD as described in the main text. (b) The same regions plotted in the parameter plane, which is roughly orthogonal to the rings. The size of the points indicates the deviation from the experimental $I_{1} / I_{2}$ ratio defined as Size $=\frac{1}{\mid I_{1} / I_{2} \text { (Calc) }-I_{1} / I_{2}(\mathrm{Obs}) \mid}$. The sketch explains the definition of the $\varphi$ angle. (c) $I_{1} / I_{2}$ as a function of $\varphi$ for each parameter region. Solid and dotted gray lines represent the observed ratio $I_{1} / I_{2}=0.83(6)$.

sidered the solutions within the interval $I_{1} / I_{2}=[0.77-0.89]$. In that case we obtained six main regions of the parameter space (two for each ring), which meet the required ratio $I_{1} / I_{2}$. Note that both INS spectra and the magnetization depend only on absolute values, but not on the sign of the $B_{4}^{4}$ coefficient; therefore, we could further reduce the number of the considered regions to three.

TABLE I. Parameters of SVD fitting for the three rings from Fig. 12(a) (all $\overline{B_{l}^{m}}$ in $\mathrm{meV}$ ).

\begin{tabular}{ccccccc}
\hline \hline & $\overline{B_{2}^{0}}$ & $\overline{B_{4}^{0}}$ & $\overline{B_{4}^{4}}$ & \multicolumn{2}{c}{ Transformation matrix } \\
\hline \multirow{2}{*}{ Red } & -0.41 & 0.022 & 0 & $\left(\begin{array}{cccc}-0.3804 & 0 & -0.0001 \\
-0.0255 & 0 & 0.0009 \\
0 & 0.1922 & 0\end{array}\right)$ \\
& & & & $\left(\begin{array}{ccc}-0.5048 & 0 & -0.0001 \\
-0.0337 & 0 & 0.001 \\
0 & 0.2623 & 0\end{array}\right)$ \\
Green & -0.161 & 0.008 & 0 & 0 \\
& & & & $\left(\begin{array}{ccc}-0.1295 & 0 & 0 \\
-0.0087 & 0 & 0.0005 \\
0 & 0.068 & 0\end{array}\right)$ \\
Blue & 0.574 & -0.03 & 0 & & & \\
& & & & & &
\end{tabular}




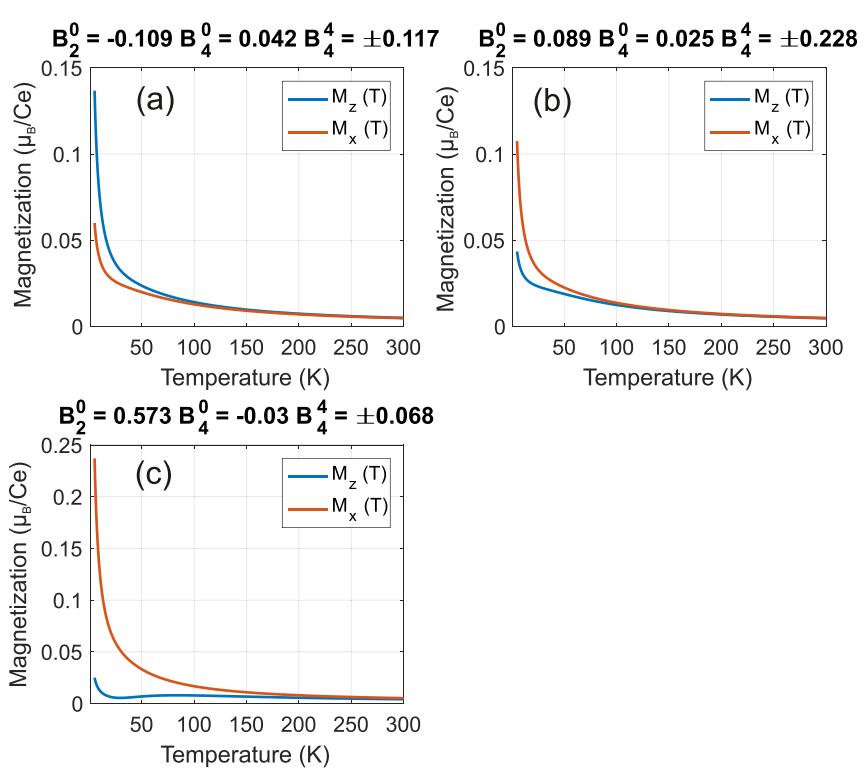

FIG. 13. Calculated temperature dependence of the magnetization for the Hamiltonian (B1). The magnetization curves were obtained for a magnetic field of $B=1 \mathrm{~T}$ applied along the $a$ and $c$ axes and three sets of $B_{l}^{m}$ parameters as indicated in each panel. $B_{l}^{m}$ parameters are given in $\mathrm{meV}$ units.

We calculated the magnetic susceptibilities along the $c$ direction and along the basal plane corresponding to the central value from each of those areas, and compared the result of these calculations with the experimental susceptibilities reported in [14]. Note that all solutions from the same parameter area result in quantitatively similar $\chi(T)$ curves. Figure 13 shows the calculated temperature dependence of the magnetization. One can see that the magnetization shown in panel (c), which corresponds to the blue ring in Fig. 12(a), exhibits a very strong easy-plane anisotropy due to the large positive $B_{2}^{0}$ coefficient. This result is in clear disagreement with the reported susceptibility data. On the other hand, two other sets of $B_{l}^{m}$ imply a relatively isotropic susceptibility, at least down to $15-20 \mathrm{~K}$. In the experiment, there was a small, but noticeable hierarchy $M_{c}>$ $M_{a}$; therefore, we believe that the first solution shown in Fig. 13(a), $B_{2}^{0}=-0.109 \mathrm{meV}, B_{4}^{0}=0.042 \mathrm{meV}$, and $B_{4}^{4}=$ $\pm 0.117 \mathrm{meV}$, provides the best fit of all experimental data.

The transition energies, wave functions. and symmetry representations of the doublets for $B_{2}^{0}=-0.109 \mathrm{meV}, B_{4}^{0}=$ $0.042 \mathrm{meV}$, and $B_{4}^{4}=0.117 \mathrm{meV}$ are given below:

$$
\begin{array}{lll}
E_{0}=0, & \psi_{0 \pm}=\mp 0.306\left| \pm \frac{5}{2}\right\rangle \pm 0.95\left|\mp \frac{3}{2}\right\rangle, & \Gamma_{7}^{(1)}, \\
E_{1}=10.78 \mathrm{meV}, & \psi_{1 \pm}=0.95\left| \pm \frac{5}{2}\right\rangle+0.306\left|\mp \frac{3}{2}\right\rangle, & \Gamma_{7}^{(2)}, \\
E_{2}=14.26 \mathrm{meV}, & \psi_{2 \pm}=1\left| \pm \frac{1}{2}\right\rangle, & \Gamma_{6} .
\end{array}
$$

Using these wave functions we calculated the magnetic moments of the ground state doublet along $c$ and $a$ directions as

$$
m_{\{\alpha=x, z\}}=g\left\langle\psi_{0}\left|J_{\alpha}\right| \psi_{0}\right\rangle,
$$

where $g=6 / 7$ for the $\mathrm{Ce}^{3+}$ ion. The moments were found to be $m_{x}=0.558 \mu_{\mathrm{B}}$ and $m_{z}=0.965 \mu_{\mathrm{B}}$.
[1] O. Stockert and F. Steglich, Unconventional quantum criticality in heavy-fermion compounds, Annu. Rev. Condens. Matter Phys. 2, 79 (2011).

[2] P. Gegenwart, Q. Si, and F. Steglich, Quantum criticality in heavy-fermion metals, Nat. Phys. 4, 186 (2008).

[3] Q. Si and F. Steglich, Heavy fermions and quantum phase transitions, Science 329, 1161 (2010).

[4] O. Stockert, J. Arndt, E. Faulhaber, C. Geibel, H. S. Jeevan, S. Kirchner, M. Loewenhaupt, K. Schmalzl, W. Schmidt, Q. Si, and F. Steglich, Magnetically driven superconductivity in $\mathrm{CeCu}_{2} \mathrm{Si}_{2}$, Nat. Phys. 7, 119 (2011).

[5] Y. Kitaoka, H. Tou, G. q. Zheng, K. Ishida, K. Asayama, T. C. Kobayashi, A. Kohda, N. Takeshita, K. Amaya, Y. Onuki, C. Geibel, C Schank, and F. Steglich, NMR study of strongly correlated electron systems, Physica B 206, 55 (1995).

[6] E. Lengyel, M. Nicklas, H. S. Jeevan, C. Geibel, and F F. Steglich, Pressure Tuning of the Interplay of Magnetism and Superconductivity in $\mathrm{CeCu}_{2} \mathrm{Si}_{2}$, Phys. Rev. Lett. 107, 057001 (2011).

[7] E. Pavarini, P. Coleman, and E. Koch, in Many-Body Physics: From Kondo to Hubbard (Theoretische Nanoelektronik, Jülich, Germany, 2015), Chap. 1.

[8] B. Shen, Y. Zhang, Y. Komijani, M. Nicklas, R. Borth, A. Wang, Y. Chen, Z. Nie, R. Li, X. Lu, H. Lee, M. Smidman, F. Steglich, P. Coleman, and H. Yuan, Strange-metal behavior in a pure ferromagnetic Kondo lattice, Nature (London) 579, 51 (2020).

[9] E. Lengyel, M. Nicklas, N. Caroca-Canales, and C. Geibel, Temperature-pressure phase diagram of CeCoSi: Pressureinduced high-temperature phase, Phys. Rev. B 88, 155137 (2013).

[10] H. Tanida, Y. Muro, and T. Matsumura, La substitution and pressure studies on $\mathrm{CeCoSi}$ : A possible antiferroquadrupolar ordering induced by pressure, J. Phys. Soc. Jpn. 87, 023705 (2018).

[11] B. Chevalier and S. F. Matar, Effect of $\mathrm{H}$ insertion on the magnetic, electronic, and structural properties of $\mathrm{CeCoSi}$, Phys. Rev. B 70, 174408 (2004).

[12] B. Chevalier, S. F. Matar, J. S. Marcos, and J. R. Fernandez, From antiferromagnetic ordering to spin fluctuation behavior induced by hydrogenation of ternary compounds $\mathrm{CeCoSi}$ and CeCoGe, Physica B 378, 795 (2006).

[13] B. Chevalier and B. Malaman, Reinvestigation of the electrical and magnetic properties of the ternary germanide $\mathrm{CeCoGe}$, Solid State Commun. 130, 711 (2004).

[14] H. Tanida, K. Mitsumoto, Y. Muro, T. Fukuhara, Y. Kawamura, A. Kondo, K. Kindo, Y. Matsumoto, T. Namiki, T. Kuwai, and T. Matsumura, Successive phase transition at ambient pressure in CeCoSi: Single crystal studies, J. Phys. Soc. Jpn. 88, 054716 (2019). 
[15] M. Manago, H. Kotegawa, H. Tou, and H. Tanida, NMR evidence of a non-magnetic phase transition in CeCoSi, J-Phys. Ann. Meet. FY2019 2019, P05 (2019).

[16] K. Hattori, Meta-orbital transition in heavy-fermion systems: Analysis by dynamical mean field theory and self-consistent renormalization theory of orbital fluctuations, J. Phys. Soc. Jpn. 79, 114717 (2010).

[17] L. V. Pourovskii, P. Hansmann, M. Ferrero, and A. Georges, Theoretical Prediction and Spectroscopic Fingerprints of an Orbital Transition in $\mathrm{CeCu}_{2} \mathrm{Si}_{2}$, Phys. Rev. Lett. 112, 106407 (2014).

[18] R. I. Bewley, J. W. Taylor, and S. M. Bennington, LET, a cold neutron multi-disk chopper spectrometer at ISIS, Nucl. Instrum. Methods Phys. Res. 637, 128 (2011).

[19] G. Ehlers, A. Podlesnyak, J. L. Niedziela, E. B. Iverson, and P. E. Sokol, The new cold neutron chopper spectrometer at the Spallation Neutron Source: Design and performance, Rev. Sci. Instrum. 82, 085108 (2011).

[20] G. Ehlers, A. Podlesnyak, and A. I. Kolesnikov, The cold neutron chopper spectrometer at the Spallation Neutron Source - A review of the first 8 years of operation, Rev. Sci. Instrum. 87, 093902 (2016).

[21] R. A. Forman, G. J. Piermarini, J. D. Barnett, and S. Block, Pressure measurement made by the utilization of ruby sharpline luminescence, Science 176, 284 (1972).

[22] A. Podlesnyak, M. Loguillo, G. M. Rucker, B. Haberl, R. Boehler, G. Ehlers, L. L. Daemen, D. Armitage, M. D. Frontzek, and M. Lumsden, Clamp cell with in situ pressure monitoring for low-temperature neutron scattering measurements, High Press. Res. 38, 482 (2018).

[23] Using of $E_{\mathrm{i}}>19 \mathrm{meV}$ could provide better energy range for studying the CEF excitations, but would strongly decrease the neutron flux on the sample.

[24] V. Petř́íček, M. Dušek, and L. Palatinus, Crystallographic computing system JANA2006: General features, Z. Kristallogr. Cryst. Mater. 229, 345 (2014).

[25] R. T. Azuah, L. R. Kneller, Y. Qiu, P. L. W. Tregenna-Piggott, C. M. Brown, J. R. D. Copley, and R. M. Dimeo, DAVE: A comprehensive software suite for the reduction, visualization, and analysis of low energy neutron spectroscopic data, J. Res. Natl. Inst. Stand. Technol. 114, 341 (2009).

[26] O. Arnold, J. C. Bilheux, J. M. Borreguero, A. Buts, S. I. Campbell, L. Chapon, M. Doucet, N. Draper, R. Ferraz Leal, M. A. Gigg, V. E. Lynch, A. Markvardsen, D. J. Mikkelson, R. L. Mikkelson, R. Miller, K. Palmen, P. Parker, G. Passos, T. G. Perring, P. F. Peterson, S. Ren, M. A. Reuter, A. T. Savici, J. W. Taylor, R. J. Taylor, R. Tolchenov, W. Zhou, and J. Zikovsky, Mantid-Data analysis and visualization package for neutron scattering and $\mu \mathrm{SR}$ experiments, Nucl. Instrum. Methods Phys. Res., A 764, 156 (2014).
[27] D. Richard, M. Ferrand, and G. J. Kearley, Analysis and visualisation of neutron-scattering data, J. Neutron Res. 4, 33 (1996).

[28] We also measured spectra with higher $E_{\mathrm{i}}=67.6 \mathrm{meV}$, but no additional magnetic excitations were observed in the spectra.

[29] We also tried a classical approach proposed by A. P. Murani for $\mathrm{CeSn}_{3}$ [41], and the results are essentially identical.

[30] P. Čermák, A. Schneidewind, B. Liu, M. M. Koza, C. Franz, R. Schönmann, O. Sobolev, and C. Pfleiderer, Magnetoelastic hybrid excitations in $\mathrm{CeAuAl}_{3}$, Proc. Natl. Acad. Sci. USA 116, 6695 (2019).

[31] M. A. Continentino, S. N. de Medeiros, M. T. D. Orlando, M. B. Fontes, and E. M. Baggio-Saitovitch, Anisotropic quantum critical behavior in $\mathrm{CeCoGe}_{3-x} \mathrm{Si}_{x}$, Phys. Rev. B 64, 012404 (2001).

[32] Note that the signal at $0.6 \mathrm{GPa}$ was measured during a separate beamtime with slightly different setup, which did not allow us to subtract the background precisely. This problem causes sharp features in Fig. 8(a) and is responsible for the lower intensity of the $0.6 \mathrm{GPa}$ signal shown in Fig. 8(b).

[33] Investigation of the form factor of the excitation is not possible due to the cell phonon scattering at high $\mathbf{Q}$ above $\sim 3 \AA^{-1}$.

[34] G. Fischer and A. Herr, Mean magnetic moments of polycrystalline Ce compounds in a tetragonal crystal field, Phys. Status Solidi B 159, K23 (1990).

[35] M. J. Besnus, A. Braghta, N. Hamdaoui, and A. Meyer, A correlation between specific heat and the ratio $T_{\mathrm{K}} / T_{\mathrm{N}}$ in magnetic Kondo lattices, J. Magn. Magn. Mater. 104, 1385 (1992).

[36] C. D. Bredl, F. Steglich, and K. D. Schotte, Specific heat of concentrated Kondo systems: (La,Ce) $\mathrm{Al}_{2}$ and $\mathrm{CeAl}_{2}$, Z. Phys. B 29, 327 (1978).

[37] F. Krüger, C. J. Pedder, and A. G. Green, Fluctuation-Driven Magnetic Hard-Axis Ordering in Metallic Ferromagnets, Phys. Rev. Lett. 113, 147001 (2014).

[38] D. Hafner, B. K. Rai, J. Banda, K. Kliemt, C. Krellner, J. Sichelschmidt, E. Morosan, C. Geibel, and M. Brando, Kondo-lattice ferromagnets and their peculiar order along the magnetically hard axis determined by the crystalline electric field, Phys. Rev. B 99, 201109(R) (2019).

[39] B. R. Cooper, Magnetic Properties of Rare Earth Metals (Springer, Boston, 1972), pp. 17-80.

[40] F. Pedregosa, G. Varoquaux, A. Gramfort, V. Michel, B. Thirion, O. Grisel, M. Blondel, P. Prettenhofer, R. Weiss, V. Dubourg, J. Vanderplas, A. Passos, D. Cournapeau, M. Brucher, M. Perrot, and E. Duchesnay, Scikit-learn: Machine learning in Python, J. Mach. Learn. Res. 12, 2825 (2011).

[41] A. P. Murani, Magnetic spectral response in the intermetallic compound $\mathrm{CeSn}_{3}$, Phys. Rev. B 28, 2308 (1983). 